\title{
First nationwide survey of US integrated 6-year cardiothoracic surgical residency program directors
}

\author{
Amir H. Lebastchi, MD, ${ }^{a}$ John J. Tackett, MD, ${ }^{a}$ Michael Argenziano, MD, ${ }^{b}$ John H. Calhoon, MD, ${ }^{c}$ \\ Mario G. Gasparri, MD, ${ }^{\mathrm{d}}$ Michael E. Halkos, MD, ${ }^{\mathrm{e}}$ George L. Hicks, Jr, MD, ${ }^{\mathrm{f}}$ Mark D. Iannettoni, MD, ${ }^{\mathrm{g}}$ \\ John S. Ikonomidis, MD, ${ }^{\mathrm{h}}$ Patrick M. McCarthy, MD, ${ }^{\mathrm{i}}$ Sandra L. Starnes, MD, ${ }^{\mathrm{j}}$ Betty C. Tong, MD, ${ }^{\mathrm{k}}$ and \\ David D. Yuh, MD
}

Objective: The recently implemented integrated 6-year (I-6) format represents a significant change in cardiothoracic surgical residency training. We report the results of the first nationwide survey assessing I- 6 program directors' impressions of this new format.

\begin{abstract}
Methods: A 28-question web-based survey was distributed to program directors of all 24 Accreditation Council for Graduate Medical Education-accredited I-6 training programs in November 2013. The response rate was a robust $67 \%$.
\end{abstract}

\begin{abstract}
Results: Compared with graduates of traditional residencies, most I-6 program directors with enrolled residents believed that their graduates will be better trained $(67 \%)$, be better prepared for new technological advances $(67 \%)$, and have superior comprehension of cardiothoracic disease processes $(83 \%)$. Just as with traditional program graduates, most respondents believed their I- 6 graduates would be able to independently perform routine adult cardiac and general thoracic operations $(75 \%)$ and were equivocal on whether additional specialty training (eg, minimally invasive, heart failure, aortic) was necessary. Most respondents did not believe that less general surgical training disadvantaged I-6 residents in terms of their career $(83 \%) ; 67 \%$ of respondents would have chosen the I- 6 format for themselves if given the choice. The greater challenges in training less mature and experienced trainees and vulnerability to attrition were noted as disadvantages of the I- 6 format. Most respondents believed that I- 6 programs represent a natural evolution toward improved residency training rather than a response to declining interest among medical school graduates.
\end{abstract}

Conclusions: High satisfaction rates with the I-6 format were prevalent among I-6 program directors. However, concerns with respect to training relatively less experienced, mature trainees were evident. (J Thorac Cardiovasc Surg 2014;148:408-15)

\section{Supplemental material is available online.}

From the Section of Cardiac Surgery, ${ }^{\text {a }}$ Department of Surgery, Yale University School of Medicine, New Haven, Conn; Section of Cardiac Surgery, ${ }^{\mathrm{b}}$ New York Presbyterian-Columbia University Medical Center, New York, NY; Division of Thoracic Surgery, ${ }^{c}$ University of Texas Health Science Center, San Antonio, Tex; Division of Cardiothoracic Surgery, ${ }^{\mathrm{d}}$ Medical College of Wisconsin, Milwaukee, Wis; Division of Cardiothoracic Surgery, ${ }^{\mathrm{e}}$ Emory University School of Medicine, Atlanta, Ga; Division of Cardiothoracic Surgery, ${ }^{\mathrm{f}}$ University of Rochester Medical Center, Rochester, NY; Department of Cardiothoracic Surgery, ${ }^{\mathrm{g}}$ University of Iowa Carver College of Medicine, Iowa City, Iowa; Division of Cardiothoracic Surgery, ${ }^{\mathrm{h}}$ Medical University of South Carolina, Charleston, SC; Division of Cardiac Surgery, ${ }^{\mathrm{i}}$ Northwestern University Feinberg School of Medicine, Chicago, IIl; Division of Thoracic Surgery, ${ }^{j}$ University of Cincinnati College of Medicine, Cincinnati, Ohio; and Division of Cardiovascular and Thoracic Surgery, ${ }^{\mathrm{k}}$ Duke University School of Medicine, Durham, NC.

Disclosures: Mario G. Gasparri reports consulting fees for DePuy-Synthes. Patrick M. McCarthy reports consulting fees for Abbott and Edwards. All other authors have nothing to disclose with regard to commercial support.

Received for publication Jan 13, 2014; revisions received March 11, 2014; accepted for publication April 3, 2014; available ahead of print May 10, 2014

Address for reprints: David D. Yuh, MD, Section of Cardiac Surgery, Department of Surgery, Yale University School of Medicine, 333 Cedar St, Boardman 204, PO Box 208039, New Haven, CT 06520 (E-mail: david.yuh@yale.edu). $0022-5223 / \$ 36.00$

Copyright (c) 2014 by The American Association for Thoracic Surgery http://dx.doi.org/10.1016/j.jtcvs.2014.04.004
Since the first integrated 6-year (I-6) cardiothoracic (CT) surgical residency program was adopted at Stanford in 2007, the number of Accreditation Council for Graduate Medical Education (ACGME)-approved I-6 programs in the United States has steadily increased. This new format for CT surgical residency programs seeks (1) to attract a greater number of highly qualified trainees to the field and (2) to provide a more focused and multidisciplinary curriculum to produce $\mathrm{CT}$ surgeons better equipped to practice modern CT surgery.

Although the general perception has been that the I-6 format is moving toward achieving these objectives, current evidence has been limited and often anecdotal. In a recent survey of I-6 program applicants, Tchantchaleishvili and colleagues $^{1}$ reported that most candidates were young, high-achieving individuals oriented toward academic careers; however, the sample size was small (36 respondents, $45 \%$ response rate). Ward and colleagues ${ }^{2}$ compared the curricula between I-6 and traditional training programs. However, their study was limited in that it only considered the duration of the different rotations rather than the content (eg, case volume, experiential milestones) and noted significant curricular heterogeneity among the programs. ${ }^{2}$ 


\section{Abbreviations and Acronyms \\ ACGME $=$ Accreditation Council for Graduate Medical Education \\ $\mathrm{CT}=$ cardiothoracic \\ I-6 = integrated six-year \\ PGY = postgraduate year}

To better assess the perceived advantages, disadvantages, and concerns with the I-6 format, particularly timely given the recent graduation of the first I- 6 residents, we conducted the first nationwide survey of program directors in all US ACGME-accredited I-6 programs and report our results and interpretations.

\section{METHODS}

A 28-question, web-based electronic survey platform (Qualtrics, Provo, Utah) was distributed to the program directors of all 24 ACGME-accredited I-6 CT surgical training programs on November 1, 2013. The survey was closed December 1, 2013. Four newly accredited I-6 programs had not yet enrolled their first residents at the time of our survey. One reminder electronic mail message to the initial nonrespondents was sent, including invitations to other members of the faculty. Participation was voluntary, and the anonymity of all respondents was preserved. The survey data were exported from the platform in a pure text file format (.CSV) and subjected to basic statistical analysis. An audit of the data revealed neither duplicate responses nor multiple responses from any 1 individual.

The survey included questions specifically composed to assess the perceptions of I-6 program directors in several different areas (see Online Data Supplemental) in accordance with published data pertaining to the I-6 format $^{1-4}$ and Dr Yuh's experience as the Yale CT surgical residency program director:

Comparisons Between I-6 and Traditional Residents

Overall competence of I-6 residents and the need for additional training after graduation

Overall favorability toward the I-6 format

Perceived advantages and disadvantages of the I- 6 format

Of these questions, 25 were multiple choice and used a Likert response scale. One question contained a field for a specific numeric response, and two questions permitted free text responses. The program directors from the 4 newly accredited programs were asked to base their answers to the questions on their current knowledge and expectations of the I-6 format; these were analyzed separately. The Yale University Human Investigation Committee approved the design and conduct of the present study.

Only data from completed surveys were analyzed. GraphPad Prism software, version 6.0c (GraphPad Software, Inc, La Jolla, Calif) was used for basic statistical analysis and plotting of the data. The responses were checked for inconsistencies and errors, computed, and presented as frequencies according to the following groups:

"Active" programs: I-6 programs with currently enrolled residents

"Mature" programs: I-6 programs instituted in 2010 or earlier, with residents at or beyond the postgraduate year (PGY)3 level

"New" programs: newly accredited programs anticipating enrolling their first residents in July 2014

The free-text responses were quoted. Because we had no true control group, the results were analyzed and are presented in a descriptive manner.

\section{RESULTS}

\section{Respondents' Program Demographics}

In November 2013, there were 24 ACGME-accredited I-6 CT surgical residency programs; 20 programs had 1 to 12 residents enrolled. A $67 \%$ response rate was achieved, with 16 program directors completing the survey (Table 1). Of these respondents, $12(75 \%)$ represented "active" programs with currently enrolled residents, accounting for $69 \%$ of all I-6 residents in the United States (62 of 90). Of these active programs, a subset of 7 "mature" programs was derived, composed of programs instituted on or before 2010 (residents at or beyond the PGY4 level). Finally, 4 respondents (25\%) represented newly accredited programs anticipating enrolling their first residents in July 2014. The distribution of the programs' starting dates was as follows: 1 in 2007, 1 in 2008, 2 in 2009, 4 in 2010, 1 in 2011, 1 in 2012, 3 in 2013, and 4 in 2014.

\section{Comparisons Between Residents in I-6 and Traditional Training Programs}

Most surveyed I-6 directors of active I-6 programs believed their residents currently possessed more diagnostic and technical aptitude and academic interest than their traditional resident counterparts (Figure 1). This superiority was also anticipated at graduation. Most respondents believed that the residents' overall maturity was largely equivalent between the 2 resident groups, although $42 \%$ anticipated greater maturity among the I- 6 residents on graduation.

Compared with the graduates of traditional residencies, most directors of the active and mature I-6 programs believed their graduates will be better trained (Figure 2), be better prepared for new technological advances, and have superior comprehension of $\mathrm{CT}$ disease processes (Table 2).

\section{Overall Competence of I-6 Residents}

Among the directors of the active I- 6 programs, $75 \%$ believed their I- 6 graduates would be able to competently and independently perform routine adult cardiac and general thoracic operations. This proportion was even greater $(86 \%)$ among the directors of mature programs (Table 2). The respondents were equivocal regarding whether they thought additional specialty training (eg, minimally invasive, heart failure, aortic) was necessary for their I-6 residents but favored additional training for graduates of traditional programs.

\section{Overall Favorability Toward the I-6 Format}

Comparatively few respondents believed that most academic CT surgical faculty favored the I- 6 format over traditional programs. However, clear majorities of the directors from both active $(75 \%)$ and mature $(71 \%)$ I-6 programs 
TABLE 1. Integrated Program Director Respondents

\begin{tabular}{lcc}
\hline \multicolumn{1}{c}{ Integrated program } & $\begin{array}{c}\text { Program } \\
\text { initiation }\end{array}$ & $\begin{array}{c}\text { Residents in } \\
\text { program (n) }\end{array}$ \\
\hline Stanford University & 2007 & 12 \\
Medical University of South Carolina & 2008 & 7 \\
University of Texas Health Science & 2009 & 5 \\
$\quad$ Center at San Antonio & & 5 \\
University of Washington & 2009 & 8 \\
Columbia University & 2010 & 4 \\
Medical College of Wisconsin & 2010 & 6 \\
University of Maryland & 2010 & 4 \\
University of Rochester & 2010 & 3 \\
Northwestern University & 2011 & 4 \\
Emory University & 2012 & 2 \\
Cleveland Clinic & 2013 & 2 \\
University of Michigan & 2013 & 0 \\
Duke University & 2014 & 0 \\
University of Cincinnati & 2014 & 0 \\
University of Iowa & 2014 & 0 \\
Yale University & 2014 & \\
\hline
\end{tabular}

believed that the I-6 format represents a natural evolution toward improved residency training rather than a response to declining interest among medical school graduates (Table 2). Also, $67 \%$ of the responding directors of active programs would have chosen an I-6 program for their own training had the choice been available. Clear majorities of the respondents from active ( $83 \%$ ) and mature (71\%) I-6 programs did not believe that the reduced general surgical experience would disadvantage I-6 graduates in future career placement. Finally, respondents representing active and mature programs were largely equivocal regarding whether I-6 graduates held an advantage over traditional program graduates for new faculty positions.
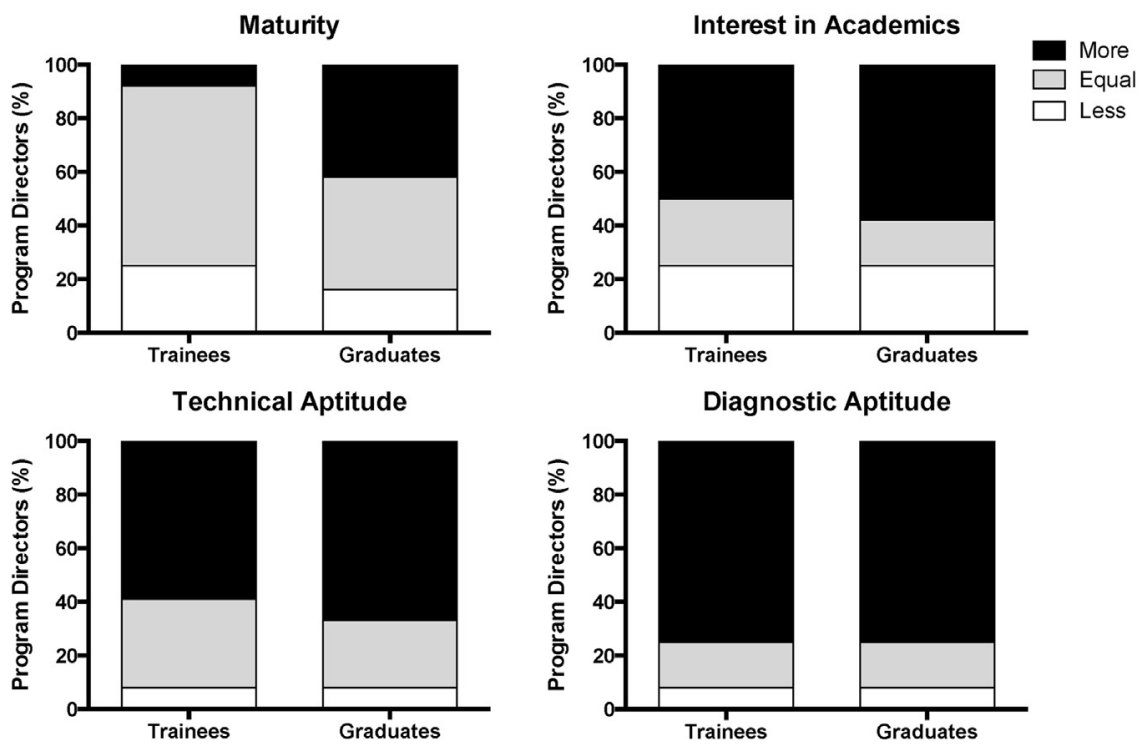

\section{Perceptions of Newly Accredited I-6 Programs}

The responses from the program directors of the 4 newly accredited I- 6 programs were tabulated and analyzed separately from the main group (Table 2), because these programs had not yet matriculated trainees at the time of the survey. These respondents were asked to anticipate their responses to the questions using their current knowledge and expectations of the new format. Although the sample size of that group was quite small, the responses suggested optimism regarding the capabilities of the I- 6 residents and features of the I- 6 format compared with those of the traditional track.

\section{Perceived Advantages and Disadvantages of the I-6 Format}

Free text responses to our questions pertaining to the perceptions of the most significant advantages and disadvantages of the I- 6 format are listed in Table 3. Prevalent advantages included the attraction of more highly qualified trainees, more time dedicated to surgical and nonsurgical training directly relevant to CT surgery, and greater opportunities for faculty mentorship. Greater challenges in training less mature and less clinically and technically experienced trainees and vulnerability to attrition were noted as disadvantages of the I-6 format.

\section{DISCUSSION}

The overarching goals of the new I- 6 training strategy are to attract a greater number of highly qualified trainees to the field and develop a more focused and relevant curriculum to produce $\mathrm{CT}$ surgeons better equipped to practice modern CT surgery. 

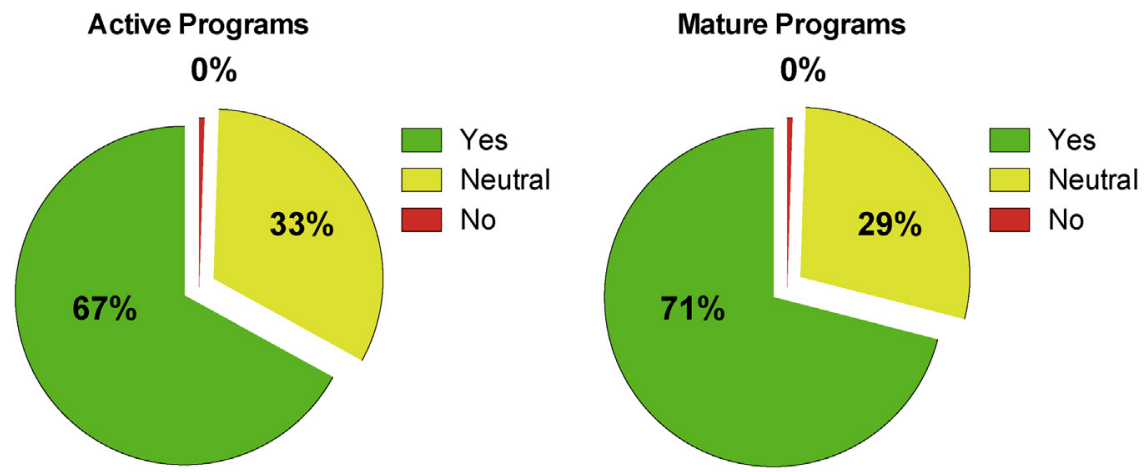

FIGURE 2. "Do you think that I-6 [6-year integrated] residency graduates will be better trained than residents who graduate from traditional residency training pathways?" Data represented as percentages of surveyed directors of 12 active and 7 mature integrated residency programs (newly accredited programs not included). Active Programs, Programs with currently enrolled residents; Mature Programs, programs initiated in 2010 or earlier (ie, with postgraduate year [PGY]4, PGY5, and PGY6 residents).

Although the realization of these goals has yet to be definitively established, the early indicators have been promising. Since the adoption of the I-6 CT surgical residency format by Stanford in 2007, steady growth has occurred in the number of ACGME-approved I-6 programs in the United States, with 14 programs offered in the 2011 to 2012 match $^{2}$ and 24 programs in the 2013 to 2014 match. Acceptance into these programs has been highly competitive, with well over 100 applications received by most programs for 1 or 2 positions each year. Furthermore, evidence has indicated that the qualifications of the I- 6 applicants have generally been superior to those of the applicants to traditional CT surgical training programs. Gasparri and colleagues $^{4}$ at the Medical College of Wisconsin and Chikwe and colleagues $^{3}$ reported a greater number of peerreviewed publications and greater US Medical Licensing Examination scores among applicants invited to interview for their I-6 program compared with their traditional program applicants. Although these parameters are not firmly established indicators for success as a surgical resident, they reflect positively on the assimilation and retention of information and interest in research.

In contrast, some concerns about the I- 6 format have arisen, including perceptions that these programs are too cardiac focused, uncertainty regarding the willingness of CT surgical faculty to train junior residents (particularly in the operating room), the heterogeneity of the curriculum among different programs, vulnerability to midcourse resident attrition, and concerns related to the lack of maturity and clinical experience otherwise obtained in traditional 5 -year general surgical training programs. ${ }^{2,4}$

The ultimate success or failure of the new I- 6 format will largely depend on whether it is perceived as achieving its primary objectives and being demonstratively superior to the tried and true traditional format. Therefore, we thought it would be useful to obtain an early snapshot of the perceptions of many of the format's architects by conducting the first nationwide survey of I-6 program directors. We have organized our discussion of the results according to several different domains.

\section{Comparison Between Residents in I-6 and Traditional Training Programs}

To date, largely anecdotal concerns have been raised that I-6 residents, generally recruited straight out of medical school, would experience significant difficulty assimilating the clinical and technical abilities relative to the fully trained general surgical residents in traditional programs. The data from our respondents suggest otherwise, indicating that their I-6 residents currently possess as much, if not more, aptitude in these areas. This finding has been substantiated, given that most respondents based their impressions on residents in their first 3 years of training.

It is possible that the lack of clinical and technical experience of I- 6 residents has been at least partially offset by the high-achieving characteristics generally ascribed to this group. From our recent nationwide survey of the I-6 residents (data not yet published), it appears that more early and intense mentorship, technical training (eg, simulation laboratories), didactics, and nontraditional rotations in cardiovascular and pulmonary rotations could also have contributed to this finding. Also, most I-6 residents appear to have had significant medical school rotational experiences in CT surgery.

Because competitive I-6 resident applicants appear to have greater numbers of research publications than traditional program applicants, it was not surprising that our surveyed program directors believed their I-6 residents currently possessed more interest in academic careers and foresaw that this would persist to graduation. A larger question, however, is how this interest in academic pursuits can be consistently fostered in I-6 programs, particularly in the basic sciences. To date, and without exception, every applicant to our I-6 program at Yale has expressed an interest and 
a desire in conducting research during their residency. It would not seem that the curricular requirements of I-6 programs, including a mandatory 24 months of general surgical rotation, would afford sufficient time to conduct highquality independent research. However, the exposure to nontraditional rotations in cardiovascular and pulmonary medicine might provide more opportunity for collaboration and new domains of investigation.

Concerns regarding the lack of overall maturity among I6 residents has been mentioned by early critics of this format. However, a clear majority of our respondents believed their I- 6 residents were as mature, if not more so, than their traditional residents, with a substantial proportion of these observing or anticipating greater degrees of maturity among their graduates. The reasons behind these observations were not clear from our survey. However, more opportunities for sustained mentorship and the discipline and focus required in academic endeavors and the other high achievements ascribed to I-6 applicants could be factors.

\section{Overall Favorability Toward the I-6 Format}

Compared with graduates of traditional residencies, most I-6 program directors believe their I- 6 residents will be better trained, better prepared for new technological advances, and have superior comprehension of CT disease processes than traditionally trained residents. Despite these perceptions, however, most I-6 program directors did not believe that the I-6 format was strongly favored over traditional programs by academic CT surgical faculty, because 41 US residency programs have not yet adopted the I- 6 approach. Whether this reflects a wait-and-see approach among most programs, the perceived disadvantages of the format, satisfaction with their own traditional programs, or the challenges in building a coalition of cardiologists, anesthesiologists, vascular surgeons, pulmonologists, and other groups not traditionally associated with CT residency training programs warrants additional elucidation beyond the scope of the present survey. Finally, a significant proportion of respondents were neutral regarding whether they believed I-6 residents would be better trained than traditionally trained residents, reflecting a current uncertainty that should not be discounted.

A clear majority of I-6 program directors believed that the I-6 format represents a natural evolution toward improved residency training rather than primarily a response to declining interest among medical school graduates. Also, $67 \%$ of the responding directors from active I- 6 programs would have chosen an I-6 program for their own training had the choice been available. Furthermore, most respondents believed their I- 6 graduates would be able to independently function as adult CT surgeons and were equivocal regarding whether additional specialty training (eg, minimally invasive, heart failure, aortic) was necessary. Finally, although the respondents were equivocal regarding whether I-6 graduates had an advantage over traditional program graduates in the academic job market, a clear majority did not believe that the reduced general surgical experience would disadvantage I-6 graduates in future career placement. These impressions were largely echoed in the responses from program directors of the newly accredited I-6 programs. These findings were not surprising, given the intuitive necessity for I-6 program directors to actively advocate for these new training programs.

To gain a more balanced perspective, however, we attempted to better understand why a few respondents did not answer positively to any of these questions. Follow-up inquiries revealed that some were not convinced that the I-6 format carries evolutionary advantages over traditional formats. Some had lingering concerns that the attenuated training period would not permit I-6 residents to acquire all the skills and maturity needed to independently practice CT surgery, that most CT surgical faculty currently do not have adequate training or experience to educate interns or junior residents, and no evidence is available that this new training paradigm will be more successful in producing well-trained CT surgeons. Several respondents would not necessarily have chosen the I-6 format for themselves, because they believed their general surgical residency was a very important part of their maturation into a physiciansurgeon and that the mentors and experiences gained during that period were important elements in their evolution as CT surgeons. Finally, some respondents believed that graduating I-6 residents who ultimately pursue general thoracic and esophageal work might be disadvantaged with the reduced general surgical training, particularly in being comfortable navigating the abdomen.

\section{Perceived Advantages and Disadvantages of the I-6 Format}

The free text responses of the most significant advantages and disadvantages of the I- 6 format were useful, particularly in identifying concerns (Table 3). The attraction of more highly qualified trainees, more time dedicated to surgical and nonsurgical training directly relevant to $\mathrm{CT}$ surgery, and greater opportunities for faculty mentorship suggest that the consensus objectives of this format are largely being realized among most current programs. Perhaps more useful, however, has been the acknowledgement that greater challenges in training less clinically and technically experienced trainees and vulnerability to attrition exist even among experienced advocates of these programs. These concerns call for a need to establish prescribed pathways and methods with respect to specific clinical rotations, didactic teaching, and technical instruction (eg, simulation laboratories) with proven track records of success, particularly during the first 3 years of I- 6 programs when relative inexperience and risk of resident attrition would seem particularly acute. Practical milestones, in 
Active programs Mature programs New programs Variable

$(\mathbf{n}=\mathbf{1 2})$

$(\mathbf{n}=7)$

$(\mathbf{n}=4)$

Comparisons between residents in I-6 and traditional training programs

Do you think I-6 residency graduates will be better trained than residents who graduate from traditional residency training pathways?

Agree

Neutral

Disagree

Do you think trainees in your I-6 program will be better prepared to adopt new technological advances in CT surgery than traditionally trained residents?

Agree

Neutral

Disagree

Do you think the integrated multidisciplinary training of I-6 residency improves the overall comprehension of $\mathrm{CT}$ disease processes?

Agree

Neutral

Disagree

Overall competence of I-6 residents

Do you think that your I-6 residents will be sufficiently trained to perform routine cardiac and thoracic operations independently by the time they graduate from your program?

Agree

Neutral

Disagree

Do you think your I-6 residents should pursue further training after completing your program to improve their skills?*

Agree

Neutral

Disagree

Overall favorability toward the I-6 format

Do you believe most academic CT surgical faculty in the United States favor the I-6 CT residency format over traditional programs?

Agree

Neutral

Disagree

Do you believe I-6 CT residency programs largely represent a reaction to declining interest among medical school graduates?

Agree

Neutral

Disagree

Do you believe I-6 CT residency programs largely represent a natural evolution toward improved residency training methods

Agree

Neutral

Disagree

If you had it to do over again, would you choose an I-6 CT surgical residency training program over a more traditional track?

Agree

$75 \%(9 / 12)$

$67 \%(8 / 12)$

$33 \%(4 / 12)$

$71 \%(5 / 7)$

$50 \%(2 / 4)$

$0 \%(0 / 12)$

$29 \%(2 / 7)$

$50 \%(2 / 4)$

$0 \%(0 / 7)$

$0 \%(0 / 4)$

$67 \%(8 / 12)$

$33 \%(4 / 12)$

$57 \%(4 / 7)$

$100 \%(4 / 4)$

$0 \%(0 / 12)$

$43 \%(3 / 7)$

$0 \%(0 / 4)$

$0 \%(0 / 7)$

$0 \%(0 / 4)$

$83 \%(10 / 12)$

$17 \%(2 / 12)$

$0 \%(0 / 12)$

$86 \%(6 / 7)$

$75 \%(3 / 4)$

$14 \%(1 / 7)$

$25 \%(1 / 4)$

$0 \%(0 / 7)$

$0 \%(0 / 4)$

$25 \%(3 / 12)$

$0 \%(0 / 12)$

$86 \%(6 / 7)$

$100 \%(4 / 4)$

$14 \%(1 / 7)$

$0 \%(0 / 4)$

$0 \%(0 / 7)$

$0 \%(0 / 4)$

$25 \%(3 / 12)$

$50 \%(6 / 12)$

$29 \%(2 / 7)$

$29 \%(2 / 7)$

$50 \%(2 / 4)$

$25 \%(3 / 12)$

$43 \%(3 / 7)$

$25 \%(1 / 4)$

$25 \%(1 / 4)$

$17 \%(2 / 12)$

$14 \%(1 / 7)$

$25 \%(1 / 4)$

$58 \%(7 / 12)$

$43 \%(3 / 7)$

$50 \%(2 / 4)$

$25 \%(3 / 12)$

$43 \%(3 / 7)$

$25 \%(1 / 4)$

$25 \%(3 / 12)$

$43 \%(3 / 7)$

$100 \%(4 / 4)$

$33 \%(4 / 12)$

$14 \%(1 / 7)$

$0 \%(0 / 4)$

$42 \%(5 / 12)$

$43 \%(3 / 7)$

$0 \%(0 / 4)$

$75 \%(9 / 12)$

$71 \%(5 / 7)$

$75 \%(3 / 4)$

$17 \%(2 / 12)$

$14 \%(1 / 7)$

$0 \%(0 / 4)$

$8 \%(1 / 12)$

$14 \%(1 / 7)$

$25 \%(1 / 4)$

Neutral

Disagree

$67 \%(8 / 12)$

$71 \%(5 / 7)$

$75 \%(3 / 4)$

$25 \%(3 / 12)$

$29 \%(2 / 7)$

$0 \%(0 / 4)$

$8 \%(1 / 12)$

$0 \%(0 / 7)$

$33 \%(1 / 4)$

Do you believe that the lack of full general surgery training confers future career disadvantages for CT surgeons trained using the I- 6 pathway?

Agree

$0 \%(0 / 12)$

$0 \%(0 / 7)$

$25 \%(1 / 4)$

Neutral

$17 \%(2 / 12)$

$29 \%(2 / 7)$

$0 \%(0 / 4)$

$83 \%(10 / 12)$

$71 \%(5 / 7)$

$75 \%(3 / 4)$ 
TABLE 2. Continued

Active programs Mature programs New programs

$(\mathbf{n}=\mathbf{1 2})$

$(\mathbf{n}=7)$

$(n=4)$

Do you think that I-6 residency graduates have an advantage over peer traditional residency training program graduates for new faculty positions?

Agree

$42 \%(5 / 12)$

$50 \%(6 / 12)$

$43 \%(3 / 7)$

$0 \%(0 / 4)$

Neutral

$8 \%(1 / 12)$

$57 \%(4 / 7)$

$50 \%(2 / 4)$

Disagree

$0 \%(0 / 7)$

$50 \%(2 / 4)$

Active programs, Programs with currently enrolled residents; Mature programs, programs initiated in 2010 or earlier (ie, with PGY4, PGY5, and PGY6 residents); New programs, newly accredited programs anticipating enrolling first residents in July 2014; I-6, integrated 6-year; $C T$, cardiothoracic. *Minimally invasive: I-6 graduates, $92 \%$; traditional graduates, $50 \%$; heart failure: I-6 graduates, $58 \%$; traditional graduates, $75 \%$; aortic surgery: I- 6 graduates, $25 \%$; traditional graduates, $42 \%$.

particular given the current heterogeneity of the I-6 curricula, would also seem prudent. The Joint Council on Thoracic Surgical Education has been actively addressing some of these issues specific to I-6 curricula, including formation of an American Board of Surgery Case Requirement Task Force.

\section{Study Limitations}

In designing our survey, we believed it necessary to include enough questions that would most directly address the perceptions and controversies with the I- 6 format but to avoid so many as to discourage participation. Although we achieved an excellent $67 \%$ response rate with this strategy, we anticipated and recognized several limitations in the present study. First, given the limited number of ACGMEaccredited I-6 programs in the United States, the sample size in the present study was small. Nevertheless, the responding program directors accounted for approximately $69 \%$ of all I-6 residents nationwide in November 2013. From the current cohort of I- 6 programs, we anticipate 82 graduates, representing all currently accredited programs in 6 years, at which time a similar survey would likely yield a more complete perspective of the format's strengths and/ or deficiencies.

TABLE 3. Most significant advantages and disadvantages of integrated 6-year cardiothoracic residency programs?*

\footnotetext{
Most significant advantages

1. "Better and more engaged residents, more purpose built curriculum, more time of contact by CT faculty"

2. "The overall academic qualifications of I-6 applicants are superior compared with traditional"

3. "Clinical exposure and acquisition of skills during a 6-y period"

4. "Increased exposure and dedication to CT surgery, increased exposure to nonsurgical specialties related to CT surgery"

5. "Keeping residents interested in CT surgery engaged—less attrition during early training years and no 'bad mouthing' of CT surgery as a career by general surgery faculty"

6. "Ability to learn both traditional and new, innovative cardiovascular treatments, including hybrid approaches, over several years"

7. "Shorter training time, attracts brighter candidates"

8. "Capture and focus better candidates"

9. "Greater period to study field of CT surgery, teach them techniques pertinent to our field, become specialist in cardiovascular medicine"

10. "Better residents"

11. "More focus on CT"

12. "Attracting superior candidates and training them ourselves"
}

13. "Talent pool"

14. "Focused training, longer CT mentorship, better cardiology exposure, more consistently higher quality residents"

15. "Less wasted time"

$\frac{16 . \text { " } 6 \mathrm{y} \text { " }}{C T \text {, Cardiothoracic; } I-6 \text {, integrated 6-year. *Free text responses from all surveyed integrated 6-year residency program directors }}$

Most significant disadvantages

1. "There will be culls and mistakes made in selection"

2. "Potential need for fellowship training"

3. "No fall back position if candidate decides not to continue"

4. "There remain significant general surgery requirements that hinder the full execution of a true I-6 curriculum"

5. "Current CT faculty might not be prepared to train junior level residents"

6. "None"

7. "Maturity, technical ability, overall medical and surgical knowledge"

8. "Hard work to mentor and train, which requires mature and committed program director"

9. "Young and immature, hard to select residents, will probably train for private practice, because not doing research time"

10. "None"

11. "Lack of history"

12. "We are not used to training junior residents so their education might not be as good as we think without significant effort; no option to change to another specialty"

13. "Remains unchartered territory, and many have forgotten this was tried at a small number of programs in the 1970s and for reasons that have not been clearly discussed was not continued"

14. "Less seasoned resident trainees at beginning"

15. "They are younger and potentially could withdraw"

16. "No general surgery certificate" 
Second, the present survey was inherently biased, given that we would expect most I-6 program directors to respond favorably with respect to the I-6 format. However, we believed that maintaining anonymity would encourage objective assessments and criticisms. Also, we asked each program director to solicit their own faculty members, including any associate program directors, to participate in our survey and provide different perspectives. However, we were not able to garner sufficient responses for any meaningful conclusions. We must also recognize that traditional CT residencies, including many highly regarded, time-tested programs still constitute the clear majority of training programs in the United States. It would have been useful to survey a cohort of residency program directors who had not adopted the I-6 format, particularly for a better understanding of their reasons for not doing so. This should be remembered when interpreting the comparisons drawn between I-6 and traditional training programs by our respondents.

Third, given that the first I-6 residents graduated in 2013, the survey sample was skewed toward experience with trainees in their early years (ie, PGY1, PGY2, PGY3). Although this could be seen as an advantage in assessing the aptitude of relatively inexperienced trainees, it handicapped most of our respondents' ability to assess the finished product (ie, I-6 graduates). As of November 2013, only 9 I-6 programs had residents in their senior years (ie, PGY 4, PGY5, PGY6). Thus, we analyzed a subset of 7 of these more mature training programs and found that their responses generally paralleled those of the respondents from our main cohort of active I-6 programs.

Finally, we recognized that many of our conclusions of residents' capabilities and qualifications were based on subjective opinion and impressions. Although predictive standardized objective assessment methods for these domains have not been developed and validated for I-6 programs, such tools might be developed during this formative period.

Many of the responses prompted additional questions we would have liked to include in our survey. For example, it would have been useful to include questions to determine whether our respondents believed that I- 6 residents were more likely to graduate as competent $\mathrm{CT}$ surgeons than their traditional program counterparts. It would also have been informative to identify the motivations behind each of our respondents' decision to pursue an I-6 format, thoughts on how to improve their respective programs, and whether they intended to maintain a parallel traditional training program. Finally, we could have obtained more granularity with respect to specifically how our respondents believed I-6 residents would be "better trained" than traditional program residents.

The present survey has provided an important snapshot of I-6 program directors' impressions of the still nascent I-6 format, providing early indications regarding whether this format is achieving its goals of attracting more highly qualified trainees to the field and producing surgeons well versed in contemporary CT surgery. It has also provided insight in confirming and discounting the initial perceptions. We found that most I-6 program directors are optimistic that this new residency training scheme is moving toward achieving its primary goals, although significant concerns remain with respect to the challenges in training highly qualified, highly motivated, but relatively inexperienced, individuals. We are hopeful that the results of our initial survey will help focus subsequent surveys and contribute to the development of effective strategies to optimize and perhaps standardize I-6 curricula.

We would like to thank all integrated program directors who responded to our survey and Ms Suzanne Giannotti who provided assistance in coordinating our study.

\section{References}

1. Tchantchaleishvili V, Barrus B, Knight PA, Jones CE, Watson TJ, Hicks GL. Sixyear integrated CT surgery residency applicants: characteristics, expectations, and concerns. J Thorac Cardiovasc Surg. 2013;146:753-8.

2. Ward ST, Smith D, Andrei AC, Hicks GL Jr, Shemin RJ, Calhoon JH, et al. Comparison of cardiothoracic training curricula: integrated six-year versus traditional programs. Ann Thorac Surg. 2013;95:2051-4; discussion 2054-6.

3. Chikwe J, Brewer Z, Goldstein AB, Adams DH. Integrated thoracic residency program applicants: the best and the brightest? Ann Thorac Surg. 2011;92:1586-91.

4. Gasparri MG, Tisol WB, Masroor S. Impact of a six-year integrated thoracic surgery training program at the Medical College of Wisconsin. Ann Thorac Surg. 2012;93:592-5; discussion 596-7. 


\section{PROGRAM DIRECTOR SURVEY QUESTIONS}

1. In which year did you initiate your I-6 CT surgical residency training program?

2. Generally speaking, how would you compare your I-6 residents to residents in your past/present traditional residency program in clinical aptitude for diagnosing and treating CT disease processes?

3. Generally speaking, how would you compare your I-6 residents to residents in your past/present traditional residency program in surgical technical aptitude?

4. Generally speaking, how would you compare your I-6 residents to residents in your past/present traditional residency program in interest in academics?

5. Generally speaking, how would you compare your I-6 residents to residents in your past/present traditional residency program in overall maturity?

6. How do you anticipate your I-6 residency graduates will compare to your past/present traditional residency graduates with regard to clinical aptitude for diagnosing and treating CT disease processes?

7. How do you anticipate your I-6 residency graduates will compare to your past/present traditional residency graduates with regard to surgical technical aptitude?

8. How do you anticipate your I-6 residency graduates will compare to your past/present traditional residency graduates with regard to interest in academics (eg, teaching, research)?

9. How do you anticipate your I-6 residency graduates will compare to your past/present traditional residency graduates with regard to overall maturity?

10. Do you think that your I-6 residents will be sufficiently trained to perform routine cardiac and thoracic operations independently by the time they graduate from your program?

11. Do you think that trainees in your I-6 program will be better prepared to adopt new technological advances in CT surgery than traditionally trained residents?

12. Do you think your I-6 residents should pursue further training after completing your program to improve their skills?

13. Do you think further training is required for I-6 graduates to perform less-invasive CT surgery (including robotics)?
14. Do you think further training is required for I-6 graduates to perform surgical therapies for heart failure (eg, mechanical circulatory assist devices, cardiopulmonary transplantation)?

15. Do you think further training is required for I-6 graduates to perform aortic surgery?

16. Do you think further training is required for traditional residency graduates less-invasive CT surgery (including robotics)?

17. Do you think further training is required for traditional residency graduates in surgical therapies for heart failure (eg, mechanical circulatory assist devices, cardiopulmonary transplantation)?

18. Do you think further training is required for traditional residency graduates in aortic surgery?

19. Do you think that I-6 residency graduates have an advantage over peer traditional residency training program graduates for new faculty positions?

20. Do you believe that the lack of full general surgery training confers future career disadvantages for CT surgeons trained using the I-6 pathway?

21. Do you think the integrated multidisciplinary training of I-6 residency improves the overall comprehension of CT disease processes?

22. What do you believe are the greatest advantages of an I6 residency program?

23. What do you believe are the largest disadvantages of an I-6 residency program?

24. Do you believe that most academic CT surgical faculty in the United States favor the I-6 CT residency format over traditional programs?

25. Do you believe that I-6 CT residency programs largely represent a reaction to declining interest among medical school graduates?

26. Do you believe that I-6 CT residency programs largely represent a natural evolution toward improved residency training methods?

27. If you had it to do over again, would you choose an I-6 CT surgical residency training program over a more traditional track?

28. Generally speaking, do you think that I-6 residency graduates will be better trained than residents who graduate from traditional residency training pathways? 